\title{
Reno-protective effect of exogenous glutathione on experimentally-induced acute kidney injury in male rats.
}

Yahya Naguib ( $\sim$ yahyamn@agu.edu.bh )

Arabian Gulf University

Eman Elgizawy

Menoufia University

\section{Research Article}

Keywords: Acute kidney injury, oxidative stress, glutathione, ciprofloxacin, CYP4F1

Posted Date: December 10th, 2020

DOl: https://doi.org/10.21203/rs.3.rs-118623/v1

License: (c) (i) This work is licensed under a Creative Commons Attribution 4.0 International License.

Read Full License 


\section{Abstract}

Background: Acute kidney injury (AKI) or Acute renal failure (ARF) refers to the sudden damage or failure of the kidney within few hours or days and resulting in acute deterioration of the renal functions. If not properly treated, AKI may lead to chronic renal failure and possibly renal transplantation. The aim of the present study was to evaluate the role of exogenous glutathione (GSH) on ciprofloxacin (GFX)-induced AKI. We also studied the effect of glutathione administration on some genes of interest.

Methods: Forty male Wistar albino rats were equally divided into 4 groups. The control group received intra-peritoneal injection of distilled water for 15 consequent days. The GSH treated group received concomitant intra-peritoneal injection of distilled water and glutathione $(200 \mathrm{mg} / \mathrm{kg} /$ day $)$ for 15 consequent days. The CFX treated group received concomitant intra-peritoneal injection of distilled water and ciprofloxacin ( $800 \mathrm{mg} / \mathrm{kg} /$ day) for 15 consequent days. The CFX+GSH treated group received concomitant intra-peritoneal injection of $\mathrm{CFX}$ and $\mathrm{CSH}$ for 15 consequent days. Serum levels of creatinine, urea, cystatin C and GGT were measured. Renal CYP4F1, GPx, GSR gene expression was evaluated.

Results: Exogenous GSH had no significant effect on the kidney functions or the studied genes when compared to the control group. Treatment with CFX resulted in significant increase $(P<0.05)$ in creatinine, urea, cystatin $\mathrm{C}$ and GGT serum levels when compared to the control group. CFX treatment also significantly $(\mathrm{P}<0.05)$ down-regulated renal GPx, GSR mRNA levels, while it up-regulated renal CYP4F1, when compared to the corresponding values in the control rats. Serum levels of urea, creatinine and cystatin $\mathrm{C}$ were significantly lower $(\mathrm{P}<0.05)$ in $\mathrm{CFX}+\mathrm{GSH}$ group when compared to the CFX treated rats. There was significant up-regulation $(\mathrm{P}<0.05)$ of the renal, GPx, GSR and down-regulation of CYP4F1 mRNA levels in the CFX+GSH group when compared to the corresponding values in the CFX treated group.

Conclusion: Our results suggest a potential prophylactic and possibly therapeutic roles of exogenous GSH administration in the treatment of drug-induced AKI. We also demonstrated that the underlying mechanism could be explained, at least in part, by the antioxidant and gene modifying properties of GSH.

\section{Background}

Acute kidney injury (AKI) or acute renal failure (ARF) can be defined as the abrupt loss of kidney functions within few days with or without changes in the urine volume. AKI usually exhibit a rise in the serum creatinine as well as other kidney function parameters [1]. The incidence of AKI increased both in hospital and community settings. It is estimated that the incidence of AKI could be from 2 to 3 cases per 1000 population [2]. It was reported that around $7 \%$ of hospitalized patients and about $66 \%$ of patients in intensive care units could develop AKI. If untreated, patients with AKI are at high risk of multiple organ failure and death [3-6]. 
Ciprofloxacin (CPX), a commonly prescribed antimicrobial fluoroquinolone, can lead to AKI by causing the development of tubulointerstitial nephritis [7]. In experimental animals, CPX was reported to induce crystal nephropathy [8]. Timely intervention proved to be vital for the prevention of AKI-dependent complications [9]. Patients taking CPX were advised to stay well hydrated and to avoid urine alkalization to prevent crystalluria [10].

The generation of reactive oxygen species (ROS) is typically observed in the pathogenesis of AKI. Furthermore, ROS are considered to be the driving factors in cardiovascular diseases and diabetes mellitus, which either predispose to AKI or present as co-morbidities. Although ROS may play important physiological functions, unregulated accumulation of ROS could result in oxidative damage and perturbed organ functionality. However, enhanced ROS production in different pathophysiological conditions could overwhelm the capacity of the endogenous antioxidant reservoir [11]. Accumulating data support the idea that tubular and cellular oxidative damage is significantly linked to AKI. Animal models of renal ischemia or nephrotoxicity demonstrated both attenuation of tissue antioxidant status and progressive oxidative tissue damage $[12,13]$. Interestingly, critically ill or sepsis patients had increased blood levels of protein and lipid oxidation biomarkers which strongly correlated with inflammatory markers [14].

Even though the exact mechanism of AKI-induced overproduction of ROS is not yet fully elucidated, reducing ROS generation, and thereby oxidative damage, remains a potential therapeutic target. Antioxidants could interfere in the early stages of AKI pathogenesis, possibly by direct elimination of ROS or even the oxidant source. Reno-protective agents with antioxidant activities has been studied in various settings of renal cells, kidney tubules, and whole animal models of AKI [11, 15]. Glutathione (GSH) plays important roles in various physiological processes $[16,17]$. GSH is principally considered as an antioxidant, an immunity booster, and a detoxifier [18]. Due to its low molecular weight and the presence of specific cell membrane transporters, GSH can rapidly cross the cell membrane. This could enable exogenous GSH to act as an intracellular antioxidant [19].

Due to the complexity of AKI as a clinical disorder associated with severe morbidity and mortality, all possible preventive measures should be sought to avoid the deleterious consequences of the condition. In the present study, we explored the therapeutic potential of exogenous GSH in CPX-induced AKI rat model.

\section{Methods}

All experiments and animal care and use were approved by the Faculty of Medicine Menoufia University Ethics Committee. Experiments were conducted in adherence to the Guiding Principles in the Use and Care of Animals published by the National Institutes of Health (NIH Publication No 85-23, Revised 1996).

Forty male Wistar rats weighing 150-200 g were recruited for the present study. Rats were purchased from a local providing facility. Rats were left to acclimatized for 10 days and were given free access to normal diet and water in an air-conditioned room with a 12-hour light-dark cycles. 
Following acclimatization, rats were randomly divided (10 rats per group) into: 1 - Control group: rats received intra peritoneal injection of distilled water for 15 consecutive days, 2- GSH-treated group: rats received intra-peritoneal injection of $200 \mathrm{mg} / \mathrm{kg} /$ day GSH (Advanced Glutathione, Rex Pharma Group, Budapest, Hungary) for 15 consecutive days, 3-CFX-treated group: rats received intra-peritoneal injection of $800 \mathrm{mg} / \mathrm{kg} /$ day CFX (Ciproxin solution for infusion, Bayer plc, Reading Berkshire, United Kingdom) for 15 consecutive days, and 4- $\mathrm{CFX}+\mathrm{GSH}$-treated group: rats received concomitantly intra-peritoneal injection of CFX and GSH.

\section{Collection of Blood samples}

At the end of the experiment (after 15 days of treatment), rats were fasted overnight. The next morning rats were anaesthetised using intraperitoneal injection of sodium thiopental (STP, $60 \mathrm{mg} / \mathrm{kg}$ ). Blood was collected then via cardiac puncture. Blood samples were left to coagulate for almost 30 minutes at room temperature. Samples were then centrifuged (3000 rpm for $15 \mathrm{~min}$ ), and the serum was separated. Samples were stored at $-20^{\circ} \mathrm{C}$ for further biochemical analysis. At the end of the study, all rats were scarified by cervical dislocation.

\section{Biochemical analysis}

ELISA kits (MyBioSource Inc, San Diego, CA, USA) were used to measure the serum level of creatinine, urea, cystatin $C$ and gamma-glutamyl transferase (GGT) enzyme activities. An automatic optical reader (SUNRISE Touchscreen, TECHAN, Salzburg, Austria) was used to perform the tests.

\section{Quantitative RT-PCR (qRT-PCR)}

Immediately after scarifying the animal, the kidneys were removed and stored at -80 or freshly used for real time quantitative reverse transcription-polymerase chain reaction (RT-PCR) experiments. Gene specific primers were designed using Primer Express Software version 2.0 (Applied Biosystems, USA). Glyceraldehyde 3-phosphate dehydrogenase (GAPDH) was used as the housekeeping control gene (Table 1). RT- PCR assays were performed in duplicate for all target and housekeeping genes using Applied Biosystems 7500 FAST 96-well PCR machine (USA). To evaluate the effects of GSH on CFX-induced AKI, the mRNA expression levels of glutathione peroxidise (GPx), glutathione reductase (GSR) and cytochrome P450-family 4- subfamily F1 (CYP4F1) genes were evaluated by RT-PCR assay as described previously [20-22]. Briefly, total RNA was extracted following homogenizing frozen or fresh kidney specimens using TRI reagent (Sigma-Aldrich, UK). Reverse transcription of renal RNA was performed using high capacity RNA-to-cDNA kit (Applied Biosystems, CA, USA). Afterwards, the generated cDNA was used to measure mRNA expression for the target genes. With GAPDH serving as the endogenous housekeeping gene control, relative quantity of mRNA expression of the target gene of interest was calculated using the comparative $\mathrm{Ct}(2-\Delta \mathrm{Ct})$ method. Data were expressed as a ratio (target gene/GAPDH\%) and were shown as the mean \pm standard error of mean of at least three independent experiments. 


\section{Statistical analysis}

Origin ${ }^{\circledR}$ software was used for the statistical analysis of the data. Analyses of Variances (ANOVA) with Tukey's post hoc tests were applied following checking of data normality using Kolmogorov-Smirnov test . Results were expressed as mean \pm standard error (SE), and $p$ values $<0.05$ were considered significant.

\section{Results}

There was no significant difference $(P>0.05)$ between the serum levels of creatinine, urea, cystatin $C$ and GGT in the GSH treated group $(0.65 \pm 0.06 \mathrm{mg} / \mathrm{dl}, 4.13 \pm 1.84 \mathrm{mg} / \mathrm{dl}, 1.87 \pm 0.09 \mathrm{mg} / \mathrm{l}$, and $1.91 \pm 0.25 \mathrm{mg} / \mathrm{dl}$ respectively), when compared to the corresponding values in the control group $(0.62 \pm 0.06 \mathrm{mg} / \mathrm{dl}$, $3.22 \pm 0.55 \mathrm{mg} / \mathrm{dl}, 1.89 \pm 0.13 \mathrm{mg} / \mathrm{l}$, and $1.87 \pm 0.19 \mathrm{mg} / \mathrm{dl}$ respectively). There was no significant difference $(P>0.05)$ in the relative quantities (RQ\%) of GPx, GSR and CYP4F1 mRNA in the GSH treated group (113.27 $\pm 16.51,110.41 \pm 12.89$ and $104.83 \pm 12.37 \%$ respectively), when compared to the corresponding values in the control group (100\%) (Figures 1 and 2).

The serum levels of creatinine, urea, cystatin C and GGT in the CFX treated group $(1.86 \pm 0.17 \mathrm{mg} / \mathrm{dl}$, $26.63 \pm 2.65 \mathrm{mg} / \mathrm{dl}, 3.23 \pm 0.22 \mathrm{mg} / \mathrm{l}$, and $89.77 \pm 13.57 \mathrm{mg} / \mathrm{dl}$ respectively) were significantly higher $(P<0.05)$ when compared to the corresponding values in the control or the GSH treated groups. There was significant decrease $(P<0.05)$ in the serum levels of creatinine, urea, cystatin $C$ and GGT $(0.85 \pm 0.07$ $\mathrm{mg} / \mathrm{dl}, 5.04 \pm 1.96 \mathrm{mg} / \mathrm{dl}, 1.95 \pm 0.26 \mathrm{mg} / \mathrm{l}$, and $2.14 \pm 0.34 \mathrm{mg} / \mathrm{dl}$ respectively) in the CFX+GSH treated group when compared to the corresponding values in the CFX treated group. Although serum creatinine was still significantly higher, there was no significant difference $(P>0.05)$ in serum urea, cystatin $C$ and GGT when comparing the $\mathrm{CFX}+\mathrm{GSH}$ with the control and GSH treated groups (Figure 1).

GPx and GSR mRNA RQ\% were significantly lower, while CYP4F1 was significantly higher $(\mathrm{P}<0.05)$ in the CFX treated group $(32.39 \pm 2.91$ and $44.32 \pm 3.92,145.62 \pm 12.75 \%$ respectively), when compared to the corresponding values in the control or the GSH treated groups. There was significant up-regulation $(P<0.05)$ in the mRNA RQ\% of GPx and GSR genes, while CYP4F1 was down-regulated $(183.87 \pm 17.94$, $196.31 \pm 18.23,105.23 \pm 13.47 \%$ respectively) in the CFX+GSH treated group, when compared to the corresponding values in the CFX treated group. GPx and GSR mRNA levels were significantly higher, while there was no significant difference in CYP4F1 mRNA level when comparing the CFX+GSH treated group to the control or the GSH treated groups (Figure 2).

\section{Discussion}

AKI is clinical syndrome with unfavourable outcomes on a substantial number of hospitalized and nonhospitalized patients. AKI can be defined as a rapid (usually within hours to 7 days) decrease in the renal functions. AKI comprises structural damage as well as loss of function, with ill-defined single clear pathophysiology [23]. Although most of the reported cases of CFX-induced ARF occur in older patients 
with pre-existing renal disease or with high doses of the medication, young and healthy patients using therapeutic doses of the CFX could still be at risk $[24,25]$. Consequently, the impact of CFX on the kidney functions remain an important area of research. This study was an attempt to explore the potential therapeutic effect of exogenous GSH in CFX-induced AKI.

In the present study, CFX treatment resulted in rise in serum levels of creatinine and urea, which was in agreement with previously published reports [26]. Elevated levels of serum creatinine is commonly associated with elevated levels of blood urea nitrogen (BUN), and could reflect a substantial reduction in the glomerular filtration rate (GFR) [27]. The vulnerability of the renal tubular cells, in particular the proximal tubule cells, to CFX or other drugs toxic effects could be primarily due to their exposure to higher levels of the circulating toxins subsequent to their involvement in the glomerular filtrate concentration and reabsorption episodes. The possible underlying mechanisms include mitochondrial dysfunction, oxidative stress, altered tubular transport, or generation of ROS [26, 27]. One of the advantages of measuring cystatin $\mathrm{C}$ production in the body is the stability of its production process. Cystatin $\mathrm{C}$ production is not influenced by extra-renal conditions such as protein catabolism or diet. Additionally, unlike creatinine, it is not altered by age or muscle mass. Hence, the serum level of cystatin $C$ could be an ideal endogenous biomarker reflecting GFR status, as well as an early and accurate detector of nephropathy [28]. In the present study, CFX treatment resulted in an increased level of cystatin C, while in the CFX-GSH treated group serum cystatin $\mathrm{C}$ level was normal. It has been reported recently that cystatin $C$ level is correlated with oxidative stress status in cardiopulmonary bypass related AKI [29]. GGT is not only a prognosticator for the involvement of oxidative stress and inflammatory reactions in disease pathophysiology, but also a valid and economic detector of AKI [30, 31]. It was logic then to evaluate serum GGT levels in the studied groups. CFX treatment significantly increased GGT level, which could be countered by the concomitant treatment with GSH. Intriguingly, GGT, as part of the gamma glutamyl cycle, is reported to maintain GSH homeostasis. GGT restores GSH, and therefore during oxidative stress, GGT activity is associated with increased GSH [32].

The fact that GSH was capable to counter the deleterious effects of CFX on renal functions in the present study encouraged us to explore the effect of CFX on GSH homeostasis and relate that to AKI pathophysiology. In fact, GSH was selected for the present study due to its ROS scavenging capacity, that enables it to protect the cell both directly and indirectly [33]. The preservation of renal functions seen in the $\mathrm{CFX}+\mathrm{GSH}$ treated rats, marked by normal serum creatinine and urea levels, could then be explained, at least in part, by GSH antioxidant properties.

We studied then the gene expression of GPx and GSR, GSH homeostasis related gene, to test the hypothesis that exogenous GSH can activate the endogenous GSH replenishing machinery. Stimulation of GSH synthesis significantly opposed oxidative stress-induced apoptosis in human retinal pigment epithelial cells from the ARPE-19 cell line [34]. CFX induced significant decrease in both GPx and GSR mRNA genes expression, while co-treatment with GSH significantly enhanced CPx and GSR mRNA levels. GSH is a thiol compound that acts as an intracellular antioxidant. Under normal physiological conditions, reduction of GSSG by GSR producers GSH at the expense of reduced nicotinamide adenine dinucleotide 
phosphate (NADPH). Thereby, the peroxidase/glutathione reductase redox cycle is crucial for maintaining normal GSH concentration [35]. One of the explanations of fluoroquinolones side effects their tendency to produce singlet oxygen and superoxide anion. ROS have detrimental effects on NDA, proteins and lipids, and mediate various cellular processes that lead to cell death [36,37]. Despite the continuous efforts of endogenous GPx and GSR, marked by initial increase in mRNA level, to eliminate the over-production of free radicals, eventually the enzymes could be depleted by CFX with subsequent cellular damage [38].

Previously published data demonstrated that the administration of CFX in patients with urinary tract infection resulted in excessive ROS generation via CYP450-mediated metabolism with subsequent substantial attenuation of the antioxidants defence mechanism [39]. It has been shown that the induction of P450s by phenobarbital or clofibrate in mice resulted in increased generation of ROS parameters in isolated liver microsomes [40]. We tested the possibility that CYP4F1, which is highly expressed in rat renal cortex, could be involved in the depletion of endogenous GSH in response to CFX. CFX treated rats had higher levels of expression of CYP4F1 mRNA, while CFX+GSH treated rats resisted CFX-mediated CYP4F1 induction. Our results support the possibility that the attenuation of the peroxidase/glutathione reductase redox cycle could be linked to induction of CYP4F1.

\section{Conclusion}

The present study demonstrated that exogenous GSH can improve CFX-induced AKI and preserve kidney functions. Our data revealed that oxidative stress could be the underlying mechanism in nephrotoxicity. Hence, the present study supports the significance of using exogenous GSH, or possibly similar antioxidants, as an adjuvant with CFX.

\section{Declarations}

\section{ETHICS APPROVAL AND CONSENT TO PARTICIPATE}

This study was approved by the Ethical Committee of the Faculty of Medicine, Menoufia University, Egypt. CONSENT TO PUBLISH

Not applicable.

AVAILABILTY OF DATA AND MATERIALS

All data generated or analysed during this study are included in this published article.

\section{COMPETING INTERESTS}

No conflict of interests.

FUNDING 
This research did not receive any specific grant from funding agencies in the public, commercial, or notfor-profit sectors.

\section{AUTHORS' CONTRIBUTIONS}

Yahya M. Naguib carried out the PCR experiments, participated in the study design and coordination, analysed the results, performed the statistical analysis, and drafted the manuscript. Eman I. Algizawy carried out the animal experiments and biochemical assays and participated in the study design. All authors have read and approved the final version of the manuscript.

\section{AKNOWLEGMENTS}

Authors wish to thank the Faculty of Medicine - Menoufia University for providing most of the required facilities.

Authors would also like to acknowledge the assistance of Prof. Dr. Eman Badr and all members of the Central Lab - Faculty of Medicine - Menoufia University.

\section{Abbreviations}

Acute kidney injury failure (AKI), Acute renal failure (ARF), ciprofloxacin (CFX), cytochrome P450-family 4subfamily F1 (CYP4F1), gamma-glutamyl transferase (GGT), glutathione (GSH), glutathione peroxidase (GPx), glutathione reductase (GSR), glyceraldehyde 3-phosphate dehydrogenase (GAPDH), nicotinamide adenine dinucleotide phosphate (NADPH), reactive oxygen species (ROS), reverse transcriptionpolymerase chain reaction (RT-PCR), sodium thiopental (STP).

\section{References}

1. Kellum JA, Sileanu FE, Murugan R, Lucko N, Shaw AD, Clermont G: Classifying AKI by Urine Output versus Serum Creatinine Level. J Am Soc Nephrol 2015, 26(9):2231-2238.

2. Bjornstad EC, Muronya W, Smith ZH, Gibson K, Mottl AK, Charles A, Marshall SW, Golightly YM, Munthali CK, Gower EW: Incidence and epidemiology of acute kidney injury in a pediatric Malawian trauma cohort: a prospective observational study. BMC Nephro/ 2020, 21(1):98.

3. Parikh RV, Tan TC, Salyer AS, Auron A, Kim PS, Ku E, Go AS: Community-Based Epidemiology of Hospitalized Acute Kidney Injury. Pediatrics 2020, 146(3).

4. Foxwell DA, Pradhan S, Zouwail S, Rainer TH, Phillips AO: Epidemiology of emergency department acute kidney injury. Nephrology (Carlton) 2020, 25(6):457-466.

5. Hsu CY, McCulloch CE, Fan D, Ordonez JD, Chertow GM, Go AS: Community-based incidence of acute renal failure. Kidney Int 2007, 72(2):208-212.

6. Nash K, Hafeez A, Hou S: Hospital-acquired renal insufficiency. Am J Kidney Dis 2002, 39(5):930-936. 
7. Hootkins R, Fenves AZ, Stephens MK: Acute renal failure secondary to oral ciprofloxacin therapy: a presentation of three cases and a review of the literature. Clin Nephrol 1989, 32(2):75-78.

8. Ball P: Ciprofloxacin: an overview of adverse experiences. J Antimicrob Chemother 1986, 18 Suppl D:187-193.

9. Khan M, Ortega LM, Bagwan N, Nayer A: Crystal-induced acute kidney injury due to ciprofloxacin. $J$ Nephropatho/ 2015, 4(1):29-31.

10. Christ W, Lehnert T, Ulbrich B: Specific toxicologic aspects of the quinolones. Rev Infect Dis 1988, 10 Suppl 1:S141-146.

11. Ratliff BB, Abdulmahdi W, Pawar R, Wolin MS: Oxidant Mechanisms in Renal Injury and Disease. Antioxid Redox Signal 2016, 25(3):119-146.

12. Dennis JM, Witting PK: Protective Role for Antioxidants in Acute Kidney Disease. Nutrients 2017, 9(7):718.

13. Goli R, Mukku KK, Raju SB, Uppin MS: Acute Ciprofloxacin-Induced Crystal Nephropathy with Granulomatous Interstitial Nephritis. Indian J Nephrol 2017, 27(3):231-233.

14. Himmelfarb J, McMonagle E, Freedman S, Klenzak J, McMenamin E, Le P, Pupim LB, Ikizler TA: Oxidative Stress Is Increased in Critically III Patients with Acute Renal Failure. Journal of the American Society of Nephrology 2004, 15(9):2449.

15. Panizo N, Rubio-Navarro A, Amaro-Villalobos JM, Egido J, Moreno JA: Molecular Mechanisms and Novel Therapeutic Approaches to Rhabdomyolysis-Induced Acute Kidney Injury. Kidney Blood Press Res 2015, 40(5):520-532.

16. Pompella A, Corti A: Editorial: the changing faces of glutathione, a cellular protagonist. Front Pharmacol 2015, 6:98.

17. Pompella A, Visvikis A, Paolicchi A, De Tata V, Casini AF: The changing faces of glutathione, a cellular protagonist. Biochem Pharmacol 2003, 66(8):1499-1503.

18. Pastore A, Federici G, Bertini E, Piemonte F: Analysis of glutathione: implication in redox and detoxification. Clin Chim Acta 2003, 333(1):19-39.

19. Zhang Y, Zhang C, Du X, Zhou Y, Kong W, Lau GW, Chen G, Kohli GS, Yang L, Wang T et al: Glutathione Activates Type III Secretion System Through Vfr in Pseudomonas aeruginosa. Frontiers in Cellular and Infection Microbiology 2019, 9(164).

20. Ameen O, Yassien RI, Naguib YM: Activation of Fox01/SIRT1/RANKL/OPG pathway may underlie the therapeutic effects of resveratrol on aging-dependent male osteoporosis. BMC Musculoskelet Disord 2020, 21(1):375.

21. El Agamy DF, Naguib YM: CoQ10 ameliorates monosodium glutamate-induced alteration in detrusor activity and responsiveness in rats via anti-inflammatory, anti-oxidant and channel inhibiting mechanisms. BMC Urol 2019, 19(1):103.

22. Motawea SM, Noreldin RI, Naguib YM: Potential therapeutic effects of endothelial cells transdifferentiated from Wharton's Jelly-derived mesenchymal stem cells on altered vascular functions in 
aged diabetic rat model. Diabetol Metab Syndr 2020, 12:40.

23. Makris K, Spanou L: Acute Kidney Injury: Definition, Pathophysiology and Clinical Phenotypes. Clin Biochem Rev 2016, 37(2):85-98.

24. Abdel-Kader K, Palevsky PM: Acute kidney injury in the elderly. Clin Geriatr Med 2009, 25(3):331-358.

25. Shields RK, Anand R, Clarke LG, Paronish JA, Weirich M, Perone H, Kieserman J, Freedy H, Andrzejewski C, Bonilla H: Defining the incidence and risk factors of colistin-induced acute kidney injury by KDIGO criteria. PLoS One 2017, 12(3):e0173286-e0173286.

26. Hajji M, Jebali H, Mrad A, Blel Y, Brahmi N, Kheder R, Beji S, Fatma LB, Smaoui W, Krid M et al: Nephrotoxicity of Ciprofloxacin: Five Cases and a Review of the Literature. Drug Saf Case Rep 2018, 5(1):17-17.

27. Pazhayattil GS, Shirali AC: Drug-induced impairment of renal function. Int J Nephrol Renovasc Dis 2014, 7:457-468.

28. Murty MSN, Sharma UK, Pandey VB, Kankare SB: Serum cystatin C as a marker of renal function in detection of early acute kidney injury. Indian J Nephrol 2013, 23(3):180-183.

29. Kararmaz A, Arslantas MK, Aksu U, Ulugol H, Cinel I, Toraman F: Evaluation of acute kidney injury with oxidative stress biomarkers and Renal Resistive Index after cardiac surgery. Acta Chirurgica Belgica 2019:1-9.

30. Bo S, Gambino R, Durazzo M, Guidi S, Tiozzo E, Ghione F, Gentile L, Cassader M, Pagano GF: Associations between gamma-glutamyl transferase, metabolic abnormalities and inflammation in healthy subjects from a population-based cohort: a possible implication for oxidative stress. World $J$ Gastroenterol 2005, 11(45):7109-7117.

31. Masarogullari K, Camyar A, Garip A, Gokalp C, Hur E, Sarsik B, Sozmen E, Duman S: Urinary GGT: A cheaper predictor of acute kidney injury? European Journal of Internal Medicine 2013, 24:e66-e67.

32. Aygun F, Kirkoc R, Aygun D, Cam H: Gamma Glutamyl Transferase and Uric Acid Levels Can Be Associated with the Prognosis of Patients in the Pediatric Intensive Care Unit. Children (Basel) 2018, 5(11):147.

33. Kurutas EB: The importance of antioxidants which play the role in cellular response against oxidative/nitrosative stress: current state. Nutr J 2016, 15(1):71-71.

34. Zou X, Feng Z, Li Y, Wang Y, Wertz K, Weber P, Fu Y, Liu J: Stimulation of GSH synthesis to prevent oxidative stress-induced apoptosis by hydroxytyrosol in human retinal pigment epithelial cells: activation of Nrf2 and JNK-p62/SQSTM1 pathways. The Journal of Nutritional Biochemistry 2012, 23(8):994-1006.

35. Han C, Kim M-J, Ding D, Park H-J, White K, Walker L, Gu T, Tanokura M, Yamasoba T, Linser P et al: GSR is not essential for the maintenance of antioxidant defenses in mouse cochlea: Possible role of the thioredoxin system as a functional backup for GSR. PLOS ONE 2017, 12(7):e0180817.

36. Kohanski MA, Dwyer DJ, Collins JJ: How antibiotics kill bacteria: from targets to networks. Nat Rev Microbio/ 2010, 8(6):423-435. 
37. Albesa I, Becerra MC, Battán PC, Páez PL: Oxidative stress involved in the antibacterial action of different antibiotics. Biochemical and Biophysical Research Communications 2004, 317(2):605-609.

38. Baghaiee B, Aliparasti MR, Almasi S, Siahkuhian M, Baradaran B: Antioxidant Expression Response to Free Radicals in Active Men and Women Fallowing to a Session Incremental Exercise; Numerical Relationship Between Antioxidants and Free Radicals. Asian J Sports Med 2016, 7(2):e29901e29901.

39. Talla V, Veerareddy P: Oxidative stress induced by fluoroquinolones on treatment for complicated urinary tract infections in Indian patients. J Young Pharm 2011, 3(4):304-309.

40. Dostalek M, Hardy KD, Milne GL, Morrow JD, Chen C, Gonzalez FJ, Gu J, Ding X, Johnson DA, Johnson JA et al: Development of oxidative stress by cytochrome P450 induction in rodents is selective for barbiturates and related to loss of pyridine nucleotide-dependent protective systems. $J$ Biol Chem 2008, 283(25):17147-17157.

\section{Tables}

Table 1: Primers used for measuring the expression of GPX, GSH AND CYP4F1 genes.

\begin{tabular}{|lll|}
\hline GPx & Sense & AGTTCGGACATCAGGAGAATGGCA \\
\hline GSH & Anti-sense & TCACCATTCACCTCGCACTTCTCA \\
& Anti-sense & CAATGTAACCGGCACCCACAATAAC \\
CYP4F1 & Sense & AAACGGTTGATTTCAGAAGGCAGTT \\
& Anti-sense & ACAGCAGGTCCATGAACAGSAAAGG \\
GAPDH & Sense & TGCACCACCAACTGCTTAGC \\
& Anti-sense & GGCATGGACTGTGGTCATGAG \\
\hline
\end{tabular}

\section{Figures}



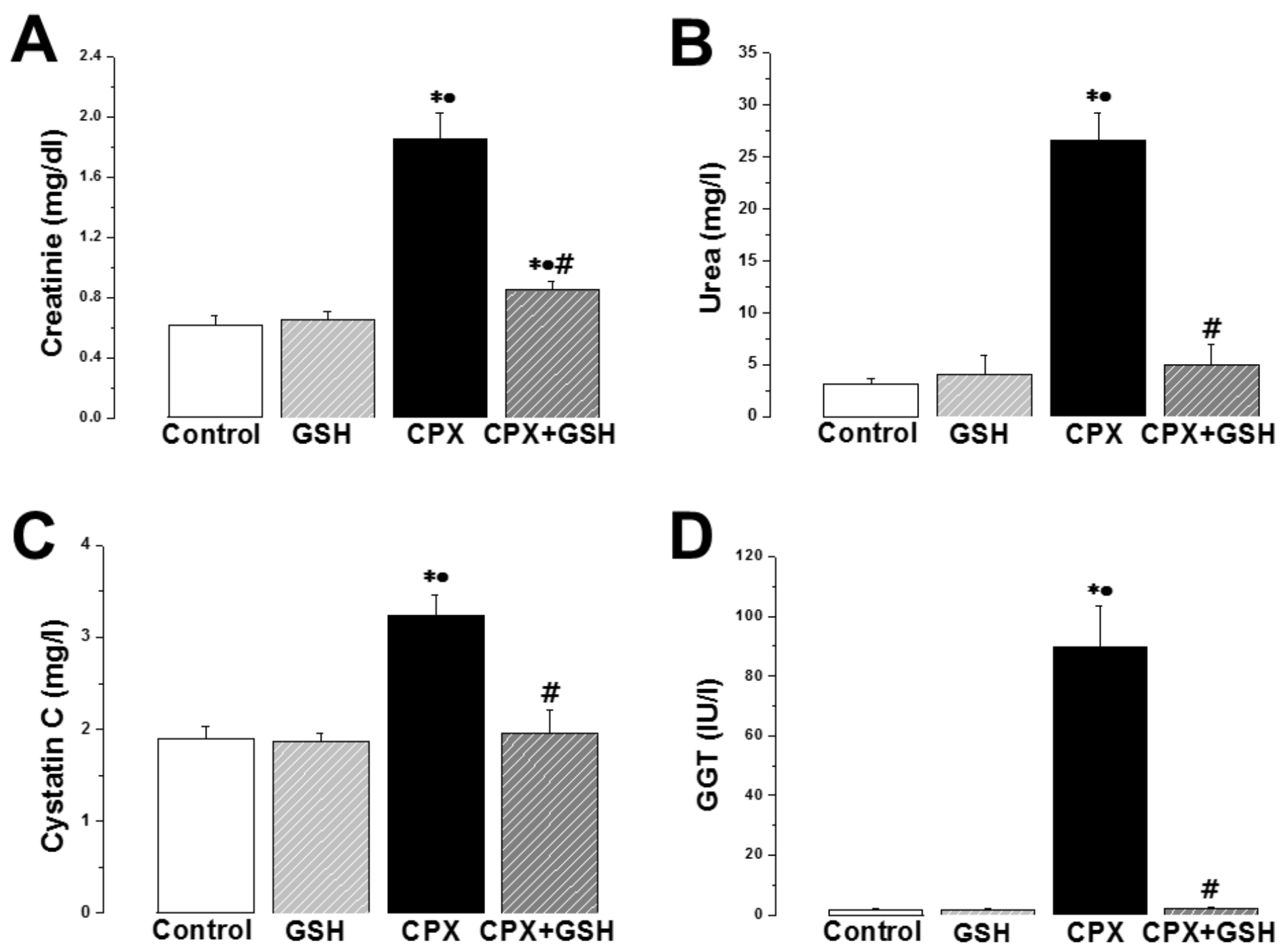

Figure 1

Exogenous GSH counters CFX-induced deterioration of kidney function. (A) Serum creatinine level in control, GFX treated, GSH treated and CFX+GSH treated groups. (B) Serum urea level in control, GFX treated, GSH treated and CFX+GSH treated groups. (C) Serum cystatin C level in control, GFX treated, GSH treated and CFX+GSH treated groups. (D) Serum GGT level in control, GFX treated, GSH treated and CFX+GSH treated groups. (Number of rats $=10 /$ group. $p<0.05$ considered significant, ${ }^{*}$ significant when compared to the control group, $\cdot$ significant when compared to the GSH group, \# significant when compared to the CFX group). 

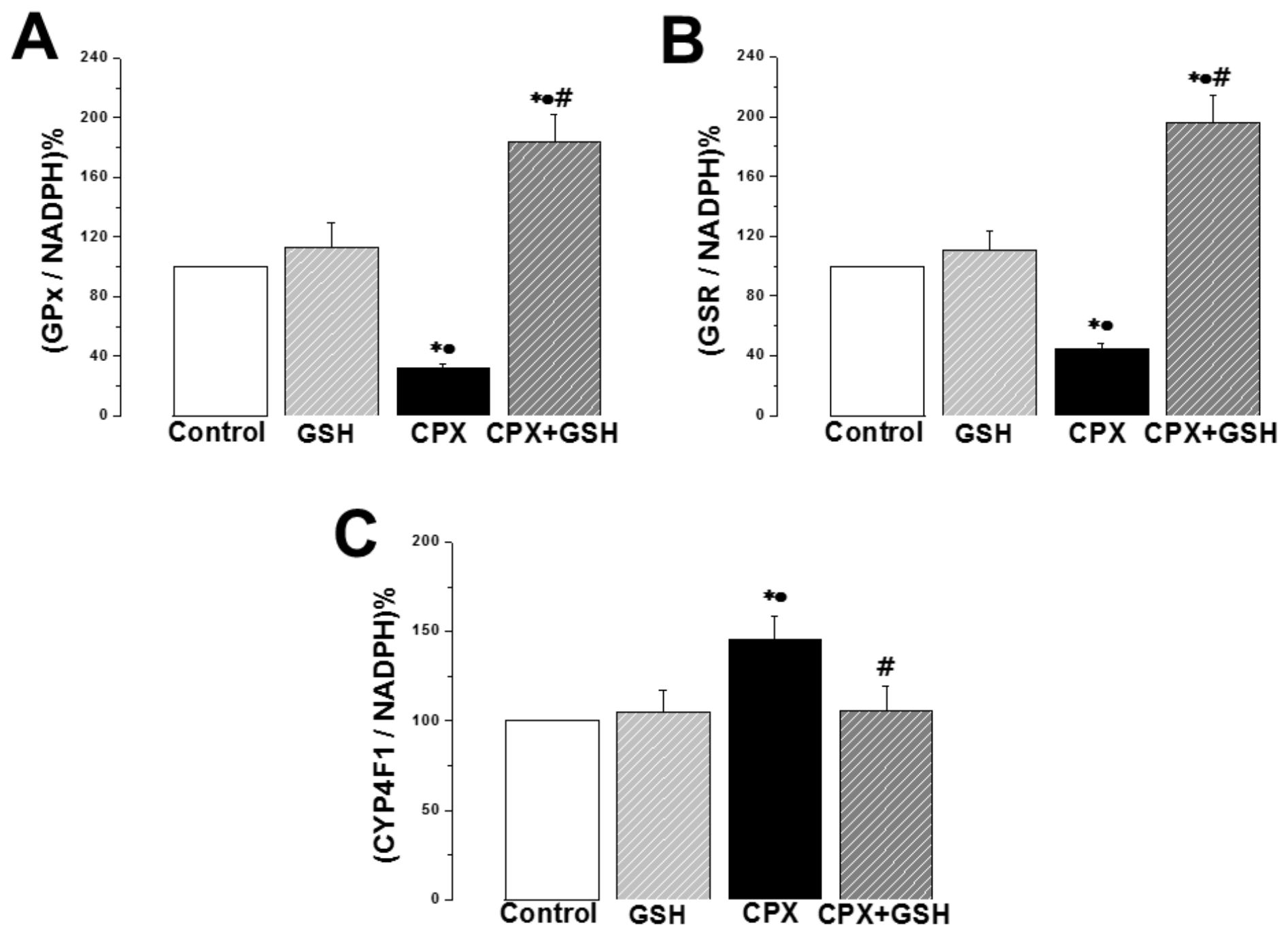

Figure 2

Effect of exogenous GSH on antioxidant enzymes gene expression. (A) GPx mRNA RQ in control, GFX treated, GSH treated and CFX+GSH treated groups. (B) GSR mRNA RQ in control, GFX treated, GSH treated and $\mathrm{CFX}+\mathrm{GSH}$ treated groups. (C) CYP4F1 mRNA RQ in control, GFX treated, GSH treated and CFX+GSH treated groups. (Number of rats $=10 /$ group. $p<0.05$ considered significant, ${ }^{*}$ significant when compared to the control group, $\cdot$ significant when compared to the GSH group, \# significant when compared to the CFX group). 